\title{
A Prospective Cohort Study to Assess Obstructive Respiratory Disease Phenotypes and Endotypes in Japan: The TRAIT Study Design
}

\author{
Nobuyuki Hizawa' \\ Koichi Fukunaga ${ }^{2}$ \\ Hisatoshi Sugiura $\mathbb{D}^{3}$ \\ Yasutaka Nakano (iD ${ }^{4}$ \\ Motokazu Kato $\mathbb{D}^{5}$ \\ Yutaro Sugiyama ${ }^{6}$ \\ Tomohiro Hanazawa ${ }^{6}$ \\ Toshihiko Kaise (1D) ${ }^{6}$ \\ Ruth Tal-Singer $\mathbb{1}^{7}$ \\ Paul W Jones $\mathbb{D}^{8}$ \\ Neil Barnes (iD $)^{8,9}$ \\ Chris Compton ${ }^{8}$ \\ Takeo Ishii ${ }^{6}$ \\ 'Department of Pulmonary Medicine, \\ Faculty of Medicine, University of \\ Tsukuba, Tsukuba, Ibaraki, Japan; \\ ${ }^{2}$ Pulmonary Division, Department of \\ Medicine, Keio University School of \\ Medicine, Tokyo, Japan; ${ }^{3}$ Department of \\ Respiratory Medicine, Tohoku University \\ Graduate School of Medicine, Sendai, \\ Japan; ${ }^{4}$ Division of Respiratory Medicine, \\ Department of Internal Medicine, Shiga \\ University of Medical Science, Shiga, \\ Japan; ${ }^{5}$ Respiratory Institute, Kamei \\ Hospital, Osaka, Japan; ${ }^{6}$ Respiratory \\ Medical Affair and Development, GSK K. \\ K., Tokyo, Japan; ${ }^{7}$ Value Evidence and \\ Outcomes, GSK, Collegeville, PA, USA \\ ${ }^{8} \mathrm{GSK}$, Brentford, Middlesex, UK; ${ }^{9}$ Barts \\ and the London School of Medicine and \\ Dentistry, London, UK
}

Correspondence: Takeo Ishii Respiratory Medical Affairs and Development, GSK K.K., Akasaka Intercity AIR, I-8-I Akasaka, Minato-ku,

Tokyo, 107-0052, Japan

Tel +8I3423। 5045

Email takeo.x.ishii@gsk.com
Background: Asthma, chronic obstructive pulmonary disease (COPD), and asthma-COPD overlap (ACO) are complex and heterogeneous diseases that share clinical characteristics (phenotypes) and molecular mechanisms (endotypes). Whilst physicians make clinical decisions on diagnostic groups, for some such as ACO there is no commonly accepted criteria. An alternative approach is to evaluate phenotypes and endotypes that are considered to respond well to a specific type of treatment ("treatable traits") rather than diagnostic labels. Purpose: The prospective, longitudinal, and observational TRAIT study will evaluate disease characteristics, including both phenotypes and endotypes, in relation to the presentation of obstructive respiratory disease characteristics in patients diagnosed with asthma, COPD, or ACO in Japan, with the aim of further understanding the clinical benefit of a treatable traits-based approach.

Patients and Methods: A total of 1500 participants will be enrolled into three cohorts according to their treating physician's diagnosis of asthma, COPD, or ACO at screening. Part 1 of the study will involve cross-sectional phenotyping and endotyping at study enrollment. Part 2 of the study will evaluate the progression of clinical characteristics, biomarker profiles, and treatment over a 3-year follow-up period. The follow-up will involve three annual study visits and three telephone calls scheduled at 6-month intervals. A substudy involving 50 participants from the asthma cohort (in which the ratio will be approximately 1:1 including 25 participants with a smoking history of $\geq 10$ pack-years and 25 participants with no smoking history), 100 participants from the ACO cohort, and 100 participants from the COPD cohort will evaluate disease phenotypes using inspiratory and expiratory computed tomography scans.

Conclusion: TRAIT will describe clinical characteristics of patients with obstructive respiratory diseases to better understand potential differences and similarities between clinical diagnoses, which will support the improvement of personalized treatment strategies. Keywords: ACO, asthma, COPD, personalized medicine, biomarkers, treatable traits

\section{Plain Language Summary}

\section{Why Was the Study Done?}

Asthma and chronic obstructive pulmonary disease (COPD) are two of the most common obstructive respiratory diseases. There are also patients in whom the features of asthma and COPD overlap and they are diagnosed with asthma-COPD overlap. These diseases are complex as they share many similar clinical and biological characteristics, often making diagnosis difficult in a precise or appropriate manner. This study looks at how we can describe the characteristics of patients with asthma, COPD, or ACO and how such 
characteristics change over time, to provide clinicians with an understanding of how they may assess and treat individual patients.

\section{What Did Researchers Do/Find?}

The study involves two parts. The first part aims to understand treatment decisions by looking at characteristics such as symptoms, comorbidities, quality of life, and biomarkers (including blood markers and imaging) among patients diagnosed with asthma, COPD, or ACO in Japan. The second part will look at how these characteristics, treatment, and disease severity change over a 3-year period.

\section{What Do These Results Mean?}

The study will increase understanding of the clinical characteristics associated with asthma, COPD, and ACO, with the aim of personalizing treatment to the individual patient to improve disease management.

\section{Introduction}

Asthma and chronic obstructive pulmonary disease (COPD) are two of the most common obstructive respiratory diseases worldwide. ${ }^{1}$ These diseases have a significant socioeconomic burden associated with them and are important drivers of impairment in health-related quality of life. $^{1-4}$ There is a large proportion of patients who present with overlapping disease characteristics and who fulfil the criteria for, and are therefore subsequently diagnosed with, asthma-COPD overlap (ACO). ${ }^{5}$ To provide context, in Japan, a recent study has reported that up to $30 \%$ of patients who presented with persistent airflow limitation shared clinical features of both asthma and COPD, and therefore fulfilled the diagnostic criteria for ACO. ${ }^{6}$ A precise definition of ACO has not yet been agreed worldwide and a review showed 11 different definitions in current use, leading to disagreement about the clinical characteristics. ${ }^{5}$ Whilst multiple criteria are used for defining ACO, there is very little concordance between patients classified using different methods. ${ }^{7}$ In 2018, the Japanese Respiratory Society (JRS) published guidelines for the management of ACO, as well as the first unified diagnostic criteria for ACO in Japan. ${ }^{6,8,9}$ ACO was defined as the coexistence of asthma features and COPD features in patients with chronic airway obstruction, with a recommendation to treat with inhaled corticosteroids (ICS) in the presence of asthma features in additional to bronchodilators. ${ }^{8}$

There has been difficulty in reaching a consensus regarding ACO due to the fact that both COPD and asthma are clinically and biologically complex diseases, therefore overlap between them increases that complexity. ${ }^{10,11}$ The typical characteristics are still under discussion, and differences exist between countries. They exhibit significant heterogeneity, sharing clinical characteristics (phenotypes) and biological mechanisms (endotypes) that are not mutually exclusive. ${ }^{10-12}$ These characteristics or traits may be present in isolation or in combination and can change over time, due to progression of the disease or as a consequence of disease management. ${ }^{13}$ Despite the heterogenous nature of these diseases, current treatment guidelines recommend a stepwise approach to disease management based on a primary diagnosis of COPD or asthma, rather than the treatable trait such as airflow obstruction or eosinophilic inflammation. ${ }^{14,15}$ Guidance on treatment for patients diagnosed with ACO is less clear, whereby Global Initiative for Chronic Obstructive Lung Disease (GOLD) guidelines no longer refer to ACO, and instead emphasize that asthma and COPD are different disorders that may share some common traits and clinical features. ${ }^{15}$ When these disorders coexist, GOLD proposes initial treatment for asthma using a stepwise approach until further investigations are performed. ${ }^{5,8,15}$ It is important to note that in the JRS guidelines, the patients should meet the criteria for COPD as well as the criteria for asthma with COPD, reflecting the fact that patients with ACO should all have COPD and that the asthma component is in addition to $\mathrm{COPD}^{8}{ }^{8}$ In clinical practice, physicians are increasingly using a personalized approach to manage patients with obstructive respiratory diseases, with treatment decisions based on clinical characteristics rather than diagnostic labels. ${ }^{15-17}$ This approach recognizes clinical characteristics that have been shown to or are considered to respond well to a specific type of treatment, referred to as "treatable traits."

The body of evidence around the overlapping clinical phenotypes, underlying biological mechanisms and management of patients across the spectrum of obstructive respiratory diseases is growing. ${ }^{18-22}$ However, there remains a need for additional large observational studies to fully characterize treatable traits. This two-part study will initially evaluate clinical characteristics, biomarker profiles, and treatment in patients diagnosed with asthma, COPD, or ACO in Japan, with the aim of better understanding treatment decisions and will go on to evaluate the progression of clinical characteristics, biomarker profiles, and treatment evolution over a 3-year longitudinal follow-up period in a Japanese population.

\section{Objectives of the Study Protocol}

The primary objective of the TRAIT study is to describe the characteristics of participants with obstructive respiratory 
diseases categorized on the basis of clinical evaluation and judgment (Part 1). Secondary objectives are to evaluate the progression of clinical characteristics over time, characterize biomarkers and profiles over time, and understand how physicians assess and treat patients with obstructive respiratory disease in clinical practice (Part 2).

\section{Materials and Methods}

\section{Study Setting and Design}

The TRAIT study (ID212311; JapicCTI-194844) ${ }^{23}$ is a prospective, longitudinal, observational cohort study, consisting of two parts (Figure 1). Participants will be enrolled into three cohorts based on a physician's diagnosis of asthma, COPD, or ACO at screening.

Part 1 of the study will involve cross-sectional phenotyping and endotyping to identify clinical and laboratory traits in participants with obstructive respiratory disease at study enrollment (Visit 1); Part 2 will evaluate the progression of clinical traits, biomarker profiles, and treatment over a 3-year, longitudinal follow-up period to identify which have the greatest predictive value for outcome and utility in clinical decision making. The followup period will involve three annual study visits and three telephone calls to participants, scheduled at 6-month intervals. Evaluable participants for Part 1 of the study will be all enrolled participants who complete Visit 1 and those for Part 2 will be all enrolled participants who complete Part 1 and at least one study visit in Part 2.

In addition, a phenotyping lung imaging substudy will be conducted to evaluate disease phenotypes through two lowdose high-resolution computed tomography scans (inspiratory and expiratory), to be performed at Visit 1 or any time in this study. A target of 50 participants from the asthma cohort (in which the ratio will be approximately 1:1 including 25 participants with a smoking history of $\geq 10$ pack-years and 25 participants with no smoking history), 100 participants from the ACO cohort, and 100 participants from the COPD cohort will be enrolled for the substudy. Participants will only be able to enter the substudy if they are enrolled at selected study sites with access to appropriate equipment. Additionally, all participants for the substudy will be required to complete at least Part 1 of this study. A steering committee including external experts and GlaxoSmithKline (GSK) guided the study design, and will guide the conduct, analysis, and interpretation.

\section{Study Population}

Participants (male or female) 40-80 years of age who are capable of giving signed, informed consent will be included into one of three cohorts based on the criteria outlined in Table 1.

In the asthma cohort at least $50 \%$ of participants must be using an ICS/long-acting $\beta_{2}$-agonist (LABA) at screening. In the ACO and COPD cohorts, at least $75 \%$ of participants must have a post-bronchodilator forced expiratory volume in 1 second/ forced vital capacity $\left(\mathrm{FEV}_{1} / \mathrm{FVC}\right)$ less than 0.7 at Visit 1, and at least $50 \%$ of participants must have experienced an event in the 24 months prior to screening, when an event is defined as symptoms of respiratory tract infection requiring antibiotics and/or systemic corticosteroids, a moderate-tosevere exacerbation, or an asthma attack. The ACO cohort will include participants who meet guideline-defined ACO, COPD with features of asthma, or asthma with features of COPD. ${ }^{9}$ Whilst participants may be enrolled into this study with a physician's diagnosis of COPD or ACO, and not meet the spirometric criteria for these conditions, the adaptive inclusion criteria will ensure that a substantial proportion of the study population meet the spirometric criterion of postbronchodilator $\mathrm{FEV}_{1} / \mathrm{FVC}$ less than 0.7. ${ }^{17}$ Screening data will be monitored to ensure the adaptive inclusion criteria
Part 1

(Cross-sectional phenotyping)
Part 2

(3-year longitudinal follow up)

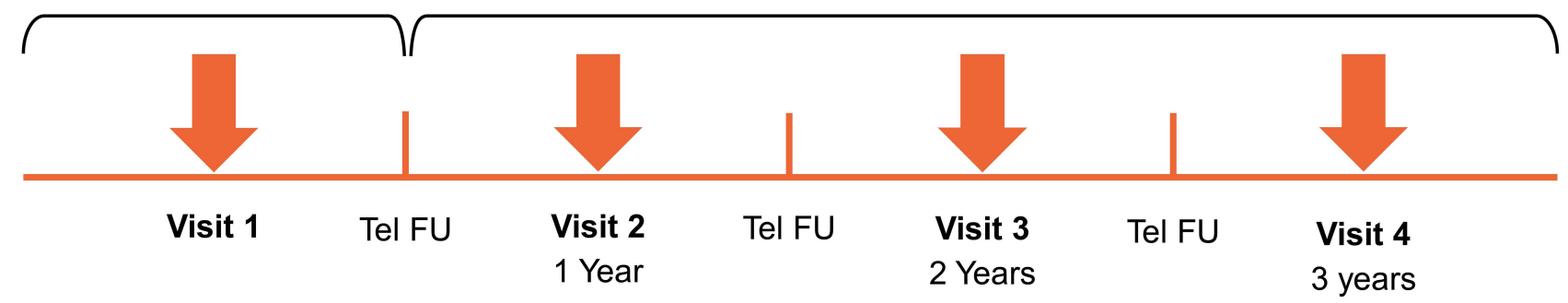

Figure I TRAIT study design.

Abbreviations: FU, follow-up; Tel, telephone. 
Table I Inclusion and Exclusion Criteria of the TRAIT Study

\begin{tabular}{|c|c|}
\hline \multicolumn{2}{|c|}{ Inclusion Criteria } \\
\hline $\begin{array}{l}\text { Asthma } \\
\text { Cohort }\end{array}$ & $\begin{array}{l}\text { A physician's diagnosis of asthma, and be a regular user of ICS maintenance medication regardless of concomitant LABA and/or } \\
\text { LAMA use }\end{array}$ \\
\hline $\begin{array}{l}\text { ACO } \\
\text { Cohort* }\end{array}$ & A physician's diagnosis of $A C O$, and be a regular user of LAMA, and/or LABA, and/or ICS maintenance medication \\
\hline $\begin{array}{l}\text { COPD } \\
\text { Cohort }\end{array}$ & $\begin{array}{l}\text { A physician's diagnosis of COPD, and be a regular user of LAMA, and/or LABA maintenance medication regardless of concomitant } \\
\text { ICS use }\end{array}$ \\
\hline \multicolumn{2}{|c|}{ Exclusion Criteria } \\
\hline & Major lung surgery (eg, lung reduction, lobectomy, or lung transplant) \\
\hline & $\begin{array}{l}\text { Known history of significant inflammatory disease other than COPD (eg, rheumatoid arthritis or lupus) (ACO and COPD } \\
\text { Cohorts only) }\end{array}$ \\
\hline & $\begin{array}{l}\text { Serious, uncontrolled disease (including serious psychological disorders) likely to interfere with the study or impact on participant } \\
\text { safety }\end{array}$ \\
\hline & Cancer diagnosis within the last 5 years (participants in remission for $\geq 5$ years are eligible) \\
\hline & Current primary diagnosis of diffuse pan-bronchiolitis, or a primary diagnosis of bronchiectasis or pulmonary fibrosis \\
\hline & Diagnosis of chronic bronchitis or pulmonary emphysema and demonstrate no spirometric evidence of obstructive lung disease \\
\hline & Participant is currently participating in another study involving investigational study treatment intervention \\
\hline & Participant is unable to perform spirometry \\
\hline
\end{tabular}

Notes: *The ACO cohort will include participants with guideline-defined ACO, COPD with features of asthma, or asthma with features of COPD.

Abbreviations: ACO, asthma-chronic obstructive pulmonary disease overlap; COPD, chronic obstructive pulmonary disease; ICS, inhaled corticosteroid; LABA, longacting $\beta_{2}$-agonist; LAMA, long-acting muscarinic antagonist.

are met. Other participants must meet the definition of unstable ACO/COPD. A participant with unstable ACO/COPD in the context of this study is defined as one who has experienced at least one of the following events in the 24 months prior to or at Visit 1: shortness of breath despite using ICS/LABA medication, symptoms (such as cough, sputum, or shortness of breath), and/or a worsening of symptoms despite using longacting muscarinic antagonist (LAMA), LAMA/LABA, or ICS/LAMA/LABA medication. No study-specific treatments will be administered; participants will continue to use medications prescribed by their regular treating physician and will continue to visit their regular treating physician for their healthcare. Data on medications taken for respiratory diseases will be collected as part of the study; however, any changes to medications during the study will be at the discretion of the participant's regular treating physician.

\section{Recruitment}

The investigators in each participating site are responsible for the recruitment of eligible patients and for following them up for three years until the end of the study. Enrollment will be closed when the number of patients reaches the target in each cohort. The adaptive criteria may restrict recruitment of patients in three categories: patients with asthma treated with ICS only (without LABA); patients with $\mathrm{ACO} / \mathrm{COPD}$ with $\mathrm{FEV}_{1} / \mathrm{FVC} \geq 0.7$ at Visit 1; patients with $\mathrm{ACO} / \mathrm{COPD}$ who experienced no acute events (defined above) for 2 years.

\section{Results}

The primary endpoint will be assessed in Part 1 of the study and is the proportion of participants presenting with different combinations of clinical characteristics (Table 2). Secondary endpoints (Table 2) will be assessed during Part 2 of the study and include clinical characteristics, biomarker measurements, and physician-assessed parameters. The endpoints of the phenotyping lung imaging substudy will include airway wall thickness, lung density, total lung capacity, and residual volume.

\section{Assessments}

The schedule for the assessment of the disease-related variables is shown in Table 3. 
Table 2 Endpoints of the TRAIT Study

\section{Primary endpoint (Part I)}

The proportion of participants presenting with combinations of clinical characteristics seen at Visit I:

- Participants with a pre-bronchodilator $\mathrm{FEV}_{1} \geq 30 \%$ and $<85 \%$, airway reversibility , at least one event $\mathrm{t}^{\mathrm{b}}$ in the 24 months prior to Visit $\mathrm{I}$, an ACQ-5 score $\geq 1.5$

- Participants with a smoking history ${ }^{\mathrm{c}}$, post-bronchodilator $\mathrm{FEV}_{\mathrm{I}} / \mathrm{FVC}<0.7$, at least one event $\mathrm{t}^{\mathrm{b}}$ in the 24 months prior to Visit $\mathrm{I}$, a CAAT ${ }^{4}$ score $\geq 10$, and peripheral blood EOS $\geq 100$ cells $/ \mu \mathrm{L}$

\section{Secondary endpoints (Part I and Part 2)}

\section{Clinical characteristics}

- The proportion of participants presenting with combinations of clinical characteristics at each visit

- The mean annual rate of events ${ }^{\mathrm{d}}$

- The mean annual rate of moderate and severe exacerbations ${ }^{\mathrm{e}}$

- Pre- and post-bronchodilator $\mathrm{FEV}_{1}, \mathrm{FVC}$, and $\mathrm{FEV} / \mathrm{FVC}$ ratio at each visit

- Airway reversibility (increase from pre-bronchodilator $\mathrm{FEV}_{1}$ of $\geq 12 \%$ and $200 \mathrm{~mL}$ ) at each visit

- CAAT, ACQ-5, SGRQ, mMRC dyspnea scale score at each visit

- Proportion of participants experiencing a clinically important deterioration in their obstructive respiratory disease per year ${ }^{f}$

- Time to lung cancer-related, respiratory-related but not lung cancer-related, and all-cause death

Biomarkers

- DLCO and FeNO at each visit

- CT scans for evaluating the degree of airways disease and emphysema

- Total and differential white blood cell count at each visit

- Proportion of participants with peripheral blood EOS count $\geq 100$ cell/ $\mu \mathrm{L}$ at each visit

- Levels of blood biomarkers* associated with respiratory disease progression and outcomes

\section{Physician-assessed parameters}

- Medical history and comorbidities at each visit

- The proportion of participants whose physician's diagnosis of asthma, COPD or ACO changes during the study

- Respiratory medications and nondrug therapies received by participants

- Type of modifications to maintenance medication for asthma/ACO/COPD during the study

- The proportion of participants who meet the GINA, 2018 definition of asthma at each visit

- The proportion of participants who meet the JRS, 2018 definition of ACO at each visit

- The proportion of participants who meet the GOLD, 2019 definition of COPD at each visit: having dyspnea, chronic cough, or sputum production, a history of exposure to risk factors, and persistent airflow limitation (post-bronchodilator $\mathrm{FEV}_{\mathrm{I}} / \mathrm{FVC}<0.7$ )

- Mean annual rate of respiratory-related HRU

- Mean annual rate of respiratory-related and all-cause hospitalizations

Notes: *Blood markers assessed will include total and differential white blood cell count; serum high-sensitivity C-reactive protein (hsCRP); serum IgE; plasma fibrinogen; serum soluble form of receptor for advanced glycation end products (sRAGE); lung matrix turnover markers; inflammatory cytokines associated with disease activity such as interleukin (IL)-17, IL-33, or chemokine ligand (CXCL)-10. aAirway reversibility is defined as follows; post-bronchodilator spirometry values show the change in $\mathrm{FEV}, \geq 12$ as a percentage on pre-bronchodilator values in combination with increase in absolute $\mathrm{FEV}, \geq 200 \mathrm{~mL}$ compared with pre-bronchodilator values. ${ }^{b} E v e n t$ includes: symptoms of respiratory tract infection requiring antibiotics and/or systemic corticosteroids, moderate-to-severe asthma/ACO/COPD exacerbation, and asthma attacks requiring additional short-acting $\beta_{2}$-agonist (SABA) treatment or SMART treatment. 'Participants with a smoking history are defined as those with a smoking history of $\geq 10$ packyears. ${ }^{\mathrm{d}}$ Event for the mean annual rate includes: symptoms of respiratory tract infection requiring antibiotics and/or systemic corticosteroids and moderate-to-severe asthma/ACO/COPD exacerbation. ${ }^{\mathrm{e}} \mathrm{A}$ moderate exacerbation is defined as a worsening of asthma/ACO/COPD that requires treatment with systemic corticosteroids and/ or antibiotics. A severe exacerbation is defined as a worsening of asthma/ACO/COPD that requires hospitalization. ${ }^{\mathrm{f}} \mathrm{A}$ clinically important deterioration is a composite measure including the worsening of key clinical features: lung function, symptoms, and exacerbation events.

Abbreviations: ACO, asthma-COPD overlap; ACQ-5, Asthma Control Questionnaire-5; CAAT, Chronic Airways Assessment Test; COPD, chronic obstructive pulmonary disease; DLCO, diffusing capacity of the lungs for carbon monoxide; EOS, eosinophil; FeNO, fractional exhaled nitric oxide; FEV ${ }_{1}$, forced expiratory volume in I second; FVC, forced vital capacity; GINA, Global Initiative for Asthma; GOLD, Global Initiative for Chronic Obstructive Lung Disease; HRU, healthcare resource utilization; JRS, Japanese Respiratory Society; mMRC, modified Medical Research Council; SGRQ, St George's Respiratory Questionnaire.

Japanese-translated versions of the following questionnaires will be completed at the start of each on-site study visit: A modified version of the COPD Assessment Test: Chronic Airways Assessment Test (CAAT), ${ }^{24}$ Asthma Control Questionnaire (ACQ-5), ${ }^{25}$ and the St George's
Respiratory Questionnaire (SGRQ). ${ }^{26}$ A summary of these questionnaires is shown in Table 4. A Japanesetranslated version of the modified Medical Research Council (mMRC) Dyspnea Scale ${ }^{27}$ will be completed by investigators and used to measure baseline functional 
Table 3 Schedule for Assessment of Disease-Related Variables

\begin{tabular}{|c|c|c|c|c|c|}
\hline \multirow[t]{2}{*}{ Variables } & \multicolumn{5}{|c|}{ Visits } \\
\hline & SCR & Visit I & Visit 2 & Visit 3 & Visit 4 \\
\hline Demography (age/gender) & $x$ & & & & \\
\hline Height, weight, and BMI & & $x$ & $\mathrm{x}$ & $\mathrm{x}$ & $x$ \\
\hline Vital signs & $x$ & $x$ & $x$ & $x$ & $x$ \\
\hline Medical history & $\mathrm{x}$ & $\mathrm{x}$ & & & \\
\hline Comorbidities & $x$ & $\mathrm{X}$ & $\mathrm{x}$ & $x$ & $\mathrm{X}$ \\
\hline Diagnosis of and medication for asthma/ACO/COPD & $\mathrm{x}$ & $\mathrm{x}$ & $\mathrm{x}$ & $\mathrm{x}$ & $\mathrm{x}$ \\
\hline Smoking status & & $x$ & $x$ & $x$ & $x$ \\
\hline Spirometry testing & & $\mathrm{x}$ & $\mathrm{X}$ & $\mathrm{X}$ & $\mathrm{x}$ \\
\hline Collect narratives of any lung CT scans* & & $x$ & $x$ & $x$ & $x$ \\
\hline Record healthcare resource utilization & & & $x$ & $x$ & $x$ \\
\hline 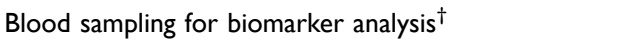 & & $x$ & $x$ & $x$ & $x$ \\
\hline DLCO and FeNO testing ${ }^{\ddagger}$ & & $x$ & $x$ & $x$ & $x$ \\
\hline CAAT, ACQ-5, SGRQ, and mMRC Dyspnea Scale & & $x$ & $x$ & $\mathrm{x}$ & $x$ \\
\hline Record occurrence of events ${ }^{\S}$ & & $x$ & $x$ & $x$ & $x$ \\
\hline
\end{tabular}

Notes: *CT scans not required as part of the main study. If a participant has had any lung CT scans performed in the year prior to entering the study or any lung CT scans performed as part of routine clinical management during the study, then the radiologist's or pulmonologist's narrative will be collected. ${ }^{\dagger} \mathrm{Blood}$ samples for analysis of emerging biomarkers will only be collected at Visit I and Visit 4. Blood samples for analysis of all other biomarkers will be collected at all study visits. ${ }^{\ddagger}$ DLCO testing and FeNO testing are only required to be performed at study sites that have access to equipment. ${ }^{\S}$ Respiratory tract infection requiring antibiotics and/or systemic corticosteroid, exacerbation, asthma attacks, serious adverse events.

Abbreviations: ACQ-5, Asthma Control Questionnaire-5; ACO, asthma-COPD overlap; BMI, body mass index; CAAT, Chronic Airways Assessment Test; COPD. Chronic obstructive pulmonary disease; CT, computerized tomography; DLCO, diffusing capacity of the lungs for carbon monoxide; FeNO, fractional exhaled nitric oxide; mMRC, modified Medical Research Council; SCR, screening; SGRQ, St George's Respiratory Questionnaire.

Table 4 Tools to Assess Symptoms in the TRAIT Study

\begin{tabular}{|c|c|c|}
\hline & Description & Scoring \\
\hline \multicolumn{3}{|c|}{ Patient-completed questionnaires } \\
\hline $\mathrm{CAAT}^{24}$ & $\begin{array}{l}\text { An 8-item questionnaire, which is a modified version of the } \\
\text { COPD Assessment Test (CAT) }{ }^{39,40} \text { with an adaptation of } \\
\text { introductory sentence referring to "chronic airway disease" } \\
\text { instead of COPD. } \\
\text { This patient-completed questionnaire covers symptoms such as } \\
\text { cough, phlegm, chest tightness, and breathlessness, and disease } \\
\text { impacts including physical activity, confidence, sleep, and energy }\end{array}$ & $\begin{array}{l}\text { Scores range from } 0 \text { to } 40 \text {, with higher scores indicating a poorer } \\
\text { chronic airway disease health status. } \\
\text { Items are scored on a } 6 \text {-point response scale, with a score of } 0 \\
\text { representing the patient not experiencing the symptom or the } \\
\text { impact at all and a score of } 5 \text { representing a maximal symptom or } \\
\text { impact. }\end{array}$ \\
\hline$A C Q-5^{25}$ & $\begin{array}{l}\text { A } 5 \text {-item questionnaire for assessing asthma control, completed } \\
\text { by the patient based on recall of the past week. Questions relate } \\
\text { to asthma symptoms }\end{array}$ & $\begin{array}{l}\text { Each item is scored on a } \\
7 \text {-point scale }(0=\text { no impairment; } 6=\text { =maximum impairment). The } \\
\text { items are equally weighted and the overall score is the mean of } \\
\text { the seven items; between } 0 \text { (well controlled) and } 6 \text { (extremely } \\
\text { poorly controlled) }\end{array}$ \\
\hline $\mathrm{SGRQ}^{26}$ & $\begin{array}{l}\text { A 76-item questionnaire used to measure health status in patients } \\
\text { with asthma and COPD }\end{array}$ & $\begin{array}{l}\text { The total score is based on three domain scores: symptoms, } \\
\text { activity, and impacts (on daily life). A decrease in score indicates } \\
\text { improvement in health status }\end{array}$ \\
\hline \multicolumn{3}{|c|}{ Investigator-completed scale } \\
\hline $\begin{array}{l}\text { mMRC } \\
\text { Dyspnea } \\
\text { Scale }^{27}\end{array}$ & $\begin{array}{l}\text { A 5-point scale used to measure baseline functional impairment } \\
\text { due to dyspnea attributable to respiratory disease by considering } \\
\text { how daily activities such as walking and climbing stairs are } \\
\text { impacted by breathlessness }\end{array}$ & $\begin{array}{l}\text { This } 5 \text {-point scale ranges from } 0=\text { breathless only with strenuous } \\
\text { exercise to } 4=\text { too breathless to leave the house }\end{array}$ \\
\hline
\end{tabular}

Abbreviations: ACQ-5, Asthma Control Questionnaire-5; CAAT, Chronic Airways Assessment Test; CAT, COPD Assessment Test; COPD, chronic obstructive pulmonary disease; mMRC, modified Medical Research Council; SGRQ, St George's Respiratory Questionnaire. 
impairment due to dyspnea attributable to respiratory disease (Table 4).

All adverse events will be monitored throughout the study and all serious adverse events will be recorded in the electronic case report form.

\section{Sample Size}

An estimated 2000 participants were intended to be screened to enroll approximately 1800 participants into the study. The rationale for the sample size is that when the proportion of participants with any individual clinical characteristic is $50 \%$, the maximum width of the $95 \%$ confidence interval (CI) is $8.2 \%$. However, due to the COVID-19 pandemic, the original sample size was revised to ensure a balance between scientific value and study feasibility. A total of 1500 participants will now be enrolled across approximately 80 sites, with 500 participants in each of the asthma, COPD, and ACO, cohorts. The maximum width of the $95 \%$ CI marginally increased to $8.9 \%$ due to the reduction of sample size.

\section{Statistical Analyses}

This is a descriptive study that is not powered to detect differences and no formal statistical hypothesis testing will be performed for any of the endpoints. A cross-sectional analysis will be performed on data from Part 1 of the study when the study is fully enrolled. The primary endpoints are the proportion of participants presenting with combinations of clinical characteristics; therefore, the percentage and $95 \%$ CI will be shown for specific combinations of characteristics. Regarding other endpoints, summary statistics will be presented. Data from Part 2 will be analyzed when all participants have completed the study. All endpoints will be explored within each of the asthma, COPD, and ACO cohorts. The change in clinical characteristics over time will be shown, which includes data from pulmonary function tests, questionnaires, and biomarkers.

\section{Discussion}

The TRAIT study will evaluate characteristics or traits, including clinical characteristics (phenotypes) and biological mechanisms (endotypes) in patients with asthma, COPD, and ACO in Japan, using a standard methodology across all three groups to investigate the different characteristics and potential clinical benefit of a treatable traitsbased approach to patient characterization.

TRAIT will provide further data to compare disease characteristics in Japan to data from large multicenter observational studies, including western cohorts and cohorts in Japan (Hokkaido, Takahata, Kyoto), which have been conducted with the aim of defining clinically relevant subtypes of COPD and asthma, as well as novel biomarkers and predictive markers of progression. ${ }^{13,18-22,24,28-30}$ These include the ECLIPSE, COPDGene, and SPIROMICS studies in COPD, and the European U-BIOPRED study in asthma. $^{13,18-22,24,31-33}$ However, whilst these studies have provided valuable insights into patient characteristics, biomarkers, and phenotypes in asthma and COPD populations, detailed characterization of ACO in these cohorts is limited due to their inclusion criteria. Individuals with ACO are more commonly excluded from studies that require a diagnosis of asthma or COPD, in part due to the lack of definitive inclusion criteria. ${ }^{9}$ The ACO registry and the NOVELTY study, however, did include individuals with ACO as part of their inclusion criteria, ${ }^{24,34}$ but more is needed to clarify the responsible molecular pathways and identify possible biomarkers for optimal therapeutic intervention in patients with ACO. ${ }^{35}$ This study will explore a different approach to classifying patients based on diagnostic labels, because it will explore the characterization of patients across the spectrum of obstructive respiratory disease using a treatable-traits approach.

\section{Strengths}

A major strength of the TRAIT study design is that it is a large prospective study across multiple different sites, enabling the longitudinal observation of obstructive lung disease in Japanese clinical practice. Another strength is the comprehensive assessment of clinical characteristics, biomarkers, and physician-assessed parameters in a large patient population across each of the three diagnostic cohorts. In this study, not only will disease-related variables be assessed at an initial baseline visit, but also longitudinally over a 3-year period. This will enable the investigators to evaluate if clinical variables, such as lung function, exacerbations, or quality of life change within the cohorts over time. In addition, the longitudinal collection of a wide variety of biomarkers, including lung imaging, to allow detection of any changes over time, with the potential that these biomarkers could be used as indicators of disease progression. Finally, the adaptive inclusion criteria allow for the recruitment of patients prescribed varying medications, in real-world clinical settings in Japan. This enables the observation of patients with relatively severe disease who require further treatment. 


\section{Limitations}

Some limitations of the TRAIT study should also be considered. First, the classification of patients into diagnostic groups will be made by physicians, using their own judgment, but whilst this may appear to be a limitation it is also a strength because it will give insights into the criteria used by clinicians in routine practice to categorize patients with airflow limitation. To accommodate this pragmatic approach to recruitment by diagnostic groups, an adaptive approach to recruitment is needed, for example to limit patients recruited to the $\mathrm{ACO}$ and COPD group who do not meet spirometric criteria for COPD. Due to the inclusion criteria, participants in the asthma cohort may experience systemic inflammatory diseases that could make it difficult to identify pathogenesis and pose a challenge when interpreting biomarkers. However, this may be mitigated by stratifying data by comorbidities during analysis. The size of the cohorts is relatively small, particularly for the substudy analysis of the high-resolution computed tomography scans, which will only involve participants enrolled at a study site that has access to appropriate equipment. The observational nature of the study also introduces limitations; participants may leave the study for different reasons and dropout rates may be different between the cohorts. The participants who remain in the study may have different characteristics to those who drop out. In addition, since this study will be conducted in Japan, the outcomes obtained may not necessarily be generalizable to other study populations in other countries. Finally, there may be a study effect in that the physicians' management of the patients may change as a result of joining the study.

\section{Clinical Implications}

Physicians worldwide, including in Japan, are increasingly choosing strategies based on the treatable-traits approach by considering the patients phenotype and endotype, rather than assigning diagnostic labels to patients and placing them within a treatment and diagnostic silo. ${ }^{13,16}$ This approach is reflected in the GOLD guidelines, which no longer refer to ACO, and emphasize that COPD and asthma are different disorders with common traits and clinical features that may be shared. However, to fully utilize this approach, potential treatable traits across the spectrum of obstructive respiratory diseases need to be fully characterized. Previous studies have demonstrated the ability to assess treatable traits in asthma and COPD, which can subsequently be targeted using a personalized-medicine approach to improve health-related quality of life, disease control, and reduce healthcare visits. ${ }^{36-38}$ Results from this study will significantly expand this knowledge base of treatable traits associated with asthma, COPD, and ACO in Japan, where this treatment approach will be important in the future. This, alongside data from other large observational studies, has the potential to advance the management of patients across the spectrum of obstructive respiratory diseases, using a personalized approach, and lead to improved patient outcomes. ${ }^{20-22,24,31}$

\section{Conclusion}

The TRAIT study will help to provide evidence to evaluate the clinical benefit of a treatable traits-based approach in patients with obstructive respiratory diseases. This in turn, will provide support for the improvement of personalized treatment strategies.

\section{Abbreviations}

ACO, asthma-COPD overlap; ACQ-5, Asthma Control Questionnaire; BMI, body mass index; CAAT, chronic airways assessment test; CI, confidence interval; COPD, chronic obstructive pulmonary disease; CT, computerized tomography; DLCO, diffusing capacity of the lungs for carbon monoxide; EOS, eosinophil; FeNO, fractional exhaled nitric oxide; $\mathrm{FEV}_{1}$, forced expiratory volume in 1 second; FU, follow-up; FVC, forced vital capacity; GINA, Global Initiative for Asthma; GOLD, Global Initiative for Chronic Obstructive Lung Disease; HRU, healthcare resource utilization; ICS, inhaled corticosteroids; JRS, Japanese Respiratory Society; LABA, longacting $\beta_{2}$-agonist; LAMA, long-acting muscarinic antagonist; mMRC, modified Medical Research Council; SCR, screening; SGRQ, St George's Respiratory Questionnaire; Tel, telephone.

\section{Ethics Approval and Informed Consent}

The study will be conducted in accordance with applicable local regulations, and the principles stated in the Declaration of Helsinki. All study documents will be reviewed and approved by institutional review boards and/or independent ethics committee(s) at all investigational sites. All patients will be asked to provide written, informed consent. 


\section{Acknowledgments}

Editorial support in the form of preparation of the first draft based on input from all authors, and collation and incorporation of author feedback to develop subsequent drafts, was provided by Hayley Mukherjee, $\mathrm{PhD}$, and Eloise Morecroft, PhD at Fishawack Indicia Ltd, UK, part of Fishawack Health, and was funded by GlaxoSmithKline (GSK). Parts of this paper were presented at the virtual 60th Annual Meeting of the Japanese Respiratory Society as a poster presentation.

\section{Author Contributions}

The authors meet criteria for authorship as recommended by the International Committee of Medical Journal Editors, take responsibility for the integrity of the work as a whole, contributed to the writing and reviewing of the manuscript, and have given final approval for the version to be published.

All authors made substantial contributions to the conception and design, acquisition of data, or analysis and interpretation of data; took part in drafting the article or revising it critically for important intellectual content; agreed to submit to the current journal; gave final approval of the version to be published; and agree to be accountable for all aspects of the work. Nobuyuki Hizawa, Koichi Fukunaga, Hisatoshi Sugiura, Yasutaka Nakano, Tomohiro Hanazawa, Neil Barnes, Chris Compton, Paul $\mathrm{W}$ Jones, and Takeo Ishii are members of the steering committee of the TRAIT study who advise on the study execution and the publications including data analyses from scientific or clinical viewpoints. Motokazu Kato, Yutaro Sugiyama, and Ruth Tal-Singer were previous members of the steering committee.

\section{Funding}

This study is funded by GSK (Study 212311). The funder of the study had a role in the study design, data analysis, data interpretation, and writing of the report.

\section{Disclosure}

This is a prospective, three-group cohort study with no study treatment intervention administered to participants. Nobuyuki Hizawa received scholarship donation (research grant) from GSK in 2018 and lecture fees from GSK, AstraZeneca, and Novartis. Koichi Fukunaga received personal fees from AstraZeneca and Boehringer Ingelheim. Hisatoshi Sugiura received lecture fees from GSK,
AstraZeneca, Novartis, and Boehringer Ingelheim. Yasutaka Nakano received personal fees from Nippon Boehringer Ingelheim, GSK, Novartis, Shionogi, Kyorin Pharmaceutical, Daiichi Sankyo, Chugai Pharmaceutical, Eli Lilly Japan, Sumitomo Dainippon Pharma, Eisai, Olympus, and AstraZeneca. Motokazu Kato received lecture fees from GSK. Yutaro Sugiyama and Tomohiro Hanazawa are GSK employees. Toshihiko Kaise, Paul W Jones, Neil Barnes, Chris Compton, and Takeo Ishii are GSK employees and hold stocks/shares in GSK. Ruth Tal-Singer was a GSK employee at the time of the study, holds stocks/shares in GSK, and received personal fees from Immunomet, Vocalis Health, Teva, and Ena Respiratory and Ena Therapeutics. The authors report no other conflicts of interest in this work.

\section{References}

1. World Health Organisation. Chronic respiratory diseases; 2020. Available from: https://www.who.int/health-topics/chronicrespiratory-diseases. Accessed June 2, 2021.

2. Iheanacho I, Zhang S, King D, Rizzo M, Ismaila AS. Economic burden of Chronic Obstructive Pulmonary Disease (COPD): a systematic literature review. Int J Chron Obstruct Pulmon Dis. 2020;15:439-460. doi:10.2147/COPD.S234942

3. Murray CJL, Barber RM, Foreman KJ, et al. Global, regional, and national disability-adjusted life years (DALYs) for 306 diseases and injuries and healthy life expectancy (HALE) for 188 countries, 1990 2013: quantifying the epidemiological transition. Lancet. 2015;386 (10009):2145-2191.

4. Forum of International Respiratory Societies. The Global Impact of Respiratory Disease - Second Edition. Sheffield, European Respiratory Society, 2017.

5. Global Initiative for Asthma. Diagnosis and initial treatment of asthma, COPD and asthma-COPD Overlap; 2017. Available from: https://ginasthma.org/wp-content/uploads/2019/11/GINA-GOLD -2017-overlap-pocket-guide-wms-2017-ACO.pdf. Accessed October 16, 2020.

6. Yamamura K, Hara J, Kobayashi T, et al. The prevalence and clinical features of asthma-COPD overlap (ACO) definitively diagnosed according to the Japanese Respiratory Society Guidelines for the Management of ACO 2018. j Med Invest. 2019;66(1.2):157-164. doi:10.2152/jmi.66.157

7. Bonten TN, Kasteleyn MJ, de Mutsert R, et al. Defining asthmaCOPD overlap syndrome: a population-based study. Eur Respir J. 2017;49(5):1602008. doi:10.1183/13993003.02008-2016

8. Japanese Respiratory Society. The JRS guidelines for the management of ACO; 2018.

9. Yanagisawa S, Ichinose $M$. Definition and diagnosis of asthma-COPD overlap (ACO). Allergol Int. 2018;67(2):172-178. doi:10.1016/j.alit.2018.01.002

10. Agusti A. The path to personalised medicine in COPD. Thorax. 2014;69(9):857-864. doi:10.1136/thoraxjnl-2014-205507

11. Bateman ED, Reddel HK, van Zyl-smit RN, Agusti A. The asthma-COPD overlap syndrome: towards a revised taxonomy of chronic airways diseases? Lancet Respir Med. 2015;3(9):719-728. doi:10.1016/S2213-2600(15)00254-4

12. Hizawa N. Clinical approaches towards asthma and chronic obstructive pulmonary disease based on the heterogeneity of disease pathogenesis. Clin Exp Allergy. 2016;46(5):678-687. doi:10.1111/ cea. 12731 
13. Pavord ID, Beasley R, Agusti A, et al. After asthma: redefining airways diseases. Lancet (London, England). 2018;391(10118):350-400.

14. Global Initiative for Asthma. Global strategy for asthma management and prevention; 2020. Available from: https://ginasthma.org/. Accessed September 26, 2020.

15. Global Initiative for Chronic Obstructive Lung Disease (GOLD). Global strategy for the diagnosis, management, and prevention of Chronic Obstructive Pulmonary Disease, 2021 report; 2021. Available from: https://goldcopd.org/gold-reports/. Accessed June 2, 2021.

16. Agusti A, Bel E, Thomas M, et al. Treatable traits: toward precision medicine of chronic airway diseases. Eur Respir J. 2016;47 (2):410-419. doi:10.1183/13993003.01359-2015

17. Hizawa N. LAMA/LABA vs ICS/LABA in the treatment of COPD in Japan based on the disease phenotypes. Int J Chron Obstruct Pulmon Dis. 2015;10:1093-1102. doi:10.2147/COPD.S72858

18. Couper D, LaVange LM, Han M, et al. Design of the subpopulations and intermediate outcomes in COPD study (SPIROMICS). Thorax. 2014;69(5):491-494. doi:10.1136/thoraxjnl-2013-203897

19. Faner R, Tal-Singer R, Riley JH, et al. Lessons from ECLIPSE: a review of COPD biomarkers. Thorax. 2014;69(7):666-672. doi:10.1136/thoraxjnl-2013-204778

20. Regan EA, Hokanson JE, Murphy JR, et al. Genetic epidemiology of COPD (COPDGene) study design. Copd. 2010;7(1):32-43. doi:10.3109/15412550903499522

21. Simpson AJ, Hekking -P-P, Shaw DE, et al. Treatable traits in the European U-BIOPRED adult asthma cohorts. Allergy. 2019;74 (2):406-411. doi:10.1111/all.13629

22. Vestbo J, Anderson W, Coxson HO, et al. Evaluation of COPD longitudinally to identify predictive surrogate end-points (ECLIPSE). Eur Respir J. 2008;31(4):869-873. doi:10.1183/09031936.00111707

23. ClinialTrials.jp. Obstructive respiratory disease phenotyping and endotyping study (TheTRAIT Study) in Japan; 2019. Available from: https://www.clinicaltrials.jp/cti-user/trial/ShowDirect.jsp? japicId=JapicCTI-194844. Accessed February 18, 2021.

24. Reddel HK, Gerhardsson de Verdier M, Agustí A, et al, Prospective observational study in patients with obstructive lung disease: NOVELTY design. ERJ Open Res. 2019;5(1):00036-2018. doi:10.1183/23120541.00036-2018

25. Juniper EF, Svensson K, Mork AC, Stahl E. Measurement properties and interpretation of three shortened versions of the asthma control questionnaire. Respir Med. 2005;99(5):553-558. doi:10.1016/j. rmed.2004.10.008

26. Jones PW, Quirk FH, Baveystock CM, Littlejohns P. A self-complete measure of health status for chronic airflow limitation. The St. George's Respiratory Questionnaire. Am Rev Respir Dis. 1992;145(6):1321-1327. doi:10.1164/ajrccm/145.6.1321

27. Mahler DA, Wells CK. Evaluation of clinical methods for rating dyspnea. Chest. 1988;93(3):580-586. doi:10.1378/chest.93.3.580

28. Rennard SI. The promise of observational studies (ECLIPSE, SPIROMICS, and COPDGene) in achieving the goal of personalized treatment of Chronic Obstructive Pulmonary Disease. Semin Respir Crit Care Med. 2015;36(4):478-490. doi:10.1055/s-0035-1555609
29. Barnes N, Ishii T, Hizawa N, et al. The distribution of blood eosinophil levels in a Japanese COPD clinical trial database and in the rest of the world. Int J Chron Obstruct Pulmon Dis. 2018;13:433-440. doi: 10.2147/COPD.S144108

30. Vestbo J, Reddel HK, Agustí A, et al. Characteristics by physician-assigned severity of asthma, asthma+COPD and COPD patients in the NOVELTY study. Eur Respir J. 2019;54(suppl 63): PA5029.

31. Rennard SI, Locantore N, Delafont B, et al. Identification of five chronic obstructive pulmonary disease subgroups with different prognoses in the ECLIPSE cohort using cluster analysis. Ann Am Thorac Soc. 2015;12(3):303-312. doi:10.1513/AnnalsATS.201403-125OC

32. Suzuki M, Makita H, Ito YM, Nagai K, Konno S, Nishimura M. Clinical features and determinants of COPD exacerbation in the Hokkaido COPD cohort study. Eur Respir J. 2014;43 (5):1289-1297. doi:10.1183/09031936.00110213

33. Takiguchi $\mathrm{H}$, Takeuchi $\mathrm{T}$, Niimi $\mathrm{K}$, et al. Proportion and clinical characteristics of non-asthmatic non-smokers among adults with airflow obstruction. PLoS One. 2018;13(5):e0196132. doi:10.1371/journal.pone.0196132

34. ClinialTrials.gov. A prospective cohort study of asthma and COPD overlap in Japanese COPD patients using the diagnostic criteria of The Japanese Respiratory Society (ACO-Registry). Available from: https://clinicaltrials.gov/ct2/show/NCT03577795. Accessed June 2, 2021.

35. Cosío BG, Dacal D. Pérez de Llano L. Asthma-COPD overlap: identification and optimal treatment. Ther Adv Respir Dis. 2018;12:1753466618805662. doi:10.1177/1753466618805662

36. McDonald VM, Hiles SA, Godbout K, et al. Treatable traits can be identified in a severe asthma registry and predict future exacerbations. Respirology (Carlton, Vic). 2019;24(1):37-47. doi:10.1111/resp.13389

37. McDonald VM, Clark VL, Cordova-Rivera L, Wark PAB, Baines KJ, Gibson PG. Targeting treatable traits in severe asthma: a randomised controlled trial. Eur Respir J. 2020;55(3). doi:10.1183/ 13993003.01509-2019

38. Pascoe S, Barnes N, Brusselle G, et al. Blood eosinophils and treatment response with triple and dual combination therapy in chronic obstructive pulmonary disease: analysis of the IMPACT trial. Lancet Respir Med. 2019;7(9):745-756. doi:10.1016/S2213-2600(19)301900

39. Jones PW, Harding G, Berry P, Wiklund I, Chen WH, Kline Leidy N. Development and first validation of the COPD Assessment Test. Eur Respir J. 2009;34(3):648-654. doi:10.1183/09031936.00102509

40. Gupta N, Pinto LM, Morogan A, Bourbeau J. The COPD assessment test: a systematic review. Eur Respir J. 2014;44(4):873-884. doi:10.1183/09031936.00025214

\section{Publish your work in this journal}

The International Journal of COPD is an international, peer-reviewed journal of therapeutics and pharmacology focusing on concise rapid reporting of clinical studies and reviews in COPD. Special focus is given to the pathophysiological processes underlying the disease, intervention programs, patient focused education, and self management protocols. This journal is indexed on PubMed Central, MedLine and CAS. The manuscript management system is completely online and includes a very quick and fair peer-review system, which is all easy to use. Visit http://www.dovepress.com/testimonials.php to read real quotes from published authors. 